\title{
Homology blocks of Plasmodium falciparum var genes and clinically distinct forms of severe malaria in a local population
}

\author{
Mary M Rorick ${ }^{1,2^{*}}$, Thomas S Rask ${ }^{3}$, Edward B Baskerville ${ }^{1}$, Karen P Day ${ }^{3}$ and Mercedes Pascual ${ }^{1,2,4}$
}

\begin{abstract}
Background: The primary target of the human immune response to the malaria parasite Plasmodium falciparum, P. falciparum erythrocyte membrane protein 1 (PfEMP1), is encoded by the members of the hyper-diverse var gene family. The parasite exhibits antigenic variation via mutually exclusive expression (switching) of the $\sim 60$ var genes within its genome. It is thought that different variants exhibit different host endothelial binding preferences that in turn result in different manifestations of disease.
\end{abstract}

Results: Var sequences comprise ancient sequence fragments, termed homology blocks (HBs), that recombine at exceedingly high rates. We use HBs to define distinct var types within a local population. We then reanalyze a dataset that contains clinical and var expression data to investigate whether the HBs allow for a description of sequence diversity corresponding to biological function, such that it improves our ability to predict disease phenotype from parasite genetics. We find that even a generic set of $\mathrm{HBs}$, which are defined for a small number of non-local parasites: capture the majority of local sequence diversity; improve our ability to predict disease severity from parasite genetics; and reveal a previously hypothesized yet previously unobserved parasite genetic basis for two forms of severe disease. We find that the expression rates of some HBs correlate more strongly with severe disease phenotypes than the expression rates of classic var DBLa tag types, and principal components of HB expression rate profiles further improve genotype-phenotype models. More specifically, within the large Kenyan dataset that is the focus of this study, we observe that HB expression differs significantly for severe versus mild disease, and for rosetting versus impaired consciousness associated severe disease. The analysis of a second much smaller dataset from Mali suggests that these HB-phenotype associations are consistent across geographically distant populations, since we find evidence suggesting that the same HB-phenotype associations characterize this population as well.

Conclusions: The distinction between rosetting versus impaired consciousness associated var genes has not been described previously, and it could have important implications for monitoring, intervention and diagnosis. Moreover, our results have the potential to illuminate the molecular mechanisms underlying the complex spectrum of severe disease phenotypes associated with malaria —an important objective given that only about $1 \%$ of P. falciparum infections result in severe disease.

Keywords: Plasmodium falciparum, Malaria, PfEMP1, Var, Severe disease, Homology block, Rosetting, Impaired consciousness, Recombination, Balancing selection

\footnotetext{
*Correspondence: mmrorick@gmail.com

${ }^{1}$ Department of Ecology and Evolutionary Biology, University of Michigan,

2019 Kraus Nat. Sci. Bldg., 830 North University Ave, Ann Arbor 48109-1048,

Michigan, USA

${ }^{2}$ Howard Hughes Medical Institute, Ann Arbor, Michigan, USA

Full list of author information is available at the end of the article
}

\section{Biomed Central}

(c) 2013 Rorick et al.; licensee BioMed Central Ltd. This is an open access article distributed under the terms of the Creative Commons Attribution License (http://creativecommons.org/licenses/by/2.0), which permits unrestricted use, distribution, and reproduction in any medium, provided the original work is properly cited. 


\section{Background}

The main target of the human immune response to $P$. falciparum is the antigenic protein $P$. falciparum erythrocyte membrane protein 1 (PfEMP1) [1], which is expressed on the surface of infected red blood cells and serves to bind host endothelial receptors. PfEMP1 is encoded by the members of the hyper-diverse var gene family, of which there are about 60 per parasite genome. These genes encode proteins that typically differ at the amino acid level by 34-55\% in the extracellular region of the protein that is the most highly conserved [2]. Var gene variants switch expression in a mutually exclusive manner over the course of an infection as a means of immune escape. It is thought that different PfEMP1 variants exhibit different binding preferences, which in turn result in different manifestations of disease (reviewed in, e.g., [3]).

Thousands of distinct var sequences exist even within small local populations. The sequences that make up an individual parasite's var repertoire typically differ from one another as much as var sequences sampled at random from the population, and in many populations there is negligible overlap between individual var repertoires [2]. The var sequence diversity that exists both within and between genomes is thought to account for the remarkable persistence and recurrence of infections within hosts. Due to variation in the domain composition of var genes, and the high levels of sequence diversity within domain families, var sequence variants cannot be reliably aligned by traditional methods. However, it is nevertheless clear that var diversity arises from a common set of ancient sequence fragments that recombine at exceedingly high rates [4-7]. In line with this, it has been shown that a relatively small set of so-called homology blocks (HBs) can describe $\sim 83 \%$ of the var sequence diversity found within a set of distantly related parasite genomes originating from diverse locations around the globe [8].

Var diversity within local populations is typically analyzed by sampling a $\sim 125$ aa sequence tag within DBL $\alpha$ subdomain 2 (e.g., [2]). The classic method to distinguish different tag types, which is used in most of the previous studies of var diversity (including $[9,10]$ ), relies on either the specific amino acid sequence (a level of diversity at which almost all sequences are distinct), or the presence/absence of short perfectly conserved motifs (e.g., the cysPoLV groups and the H3 subset, and when in combination with network based sequence analysis methods, the block-sharing groups that define A-like var genes) [11-13]. Some of these classic tag types are thought to be associated with certain disease phenotypes. One relatively consistent finding is that A-like var expression is associated with both rosetting [13-15] and severe disease [12], though not necessarily independently since it is well established that the rosetting phenotype correlates with severe disease [16-19]. Rosetting is defined as the binding of uninfected red blood cells by infected red blood cells. This phenotype can be clinically assayed at low cost, and it provides a particularly good starting point to look for genotype-phenotype associations because, rather than being determined by a multitude of parasite and/or host factors, it is thought that rosetting is directly mediated by PfEMP1 binding. Furthermore, the DBL $\alpha$ domain is thought to contain the actual site for PfEMP1 binding of uninfected cells [20], so variation within the DBL $\alpha$ tag may be expected to influence variation in the rosetting phenotype. Severe malaria has also recently been linked to particular domain cassettes that include the DBL $\alpha$ domain [21-24] — a finding that suggests a possible association between DBL $\alpha$ and disease severity, and further increases the likelihood that residues important for disease phenotype exist in the protein region encoded by DBL $\alpha$ tags. All of the above evidence, taken together with the great amounts of DBL $\alpha$ tag data presently available, makes this sequence region very attractive to study.

The most comprehensive DBL $\alpha$ tag dataset currently available was previously analyzed by Warimwe et al. $[9,10]$. It includes expressed DBL $\alpha$ tags (cDNA) and clinical data for 250 isolates from Kenya, as well as a sample of genomic DBL $\alpha$ tags for 53 isolates. This dataset supports the above mentioned association of A-like var expression with both rosetting and severe disease. Warimwe et al. also report another interesting set of patterns within this data: while A-like expression associates with one form of severe disease, impaired consciousness (IC), it does not correlate with another form of severe disease, respiratory distress (RD); additionally, while rosetting correlates with both RD and A-like var expression, it does not correlate with IC [10]. Based on these observations, Warimwe et al. conclude that two subsets of A-like var genes must exist that cause disease by very different means. They hypothesize that the subset associated with impaired consciousness causes severe disease through tissue specific sequestration, while the subset associated with rosetting causes $\mathrm{RD}$ and sometimes also IC through a non-tissue-specific mechanism; however, they were unable to identify a genetic marker that could distinguish these two subsets of var genes [10]. One possibility is that the var DBL $\alpha$ tag does not contain the differentiating factor, but another possibility is that the methods used by Warimwe et al. to distinguish different types of tag sequences did not fully capture all the functionally relevant genetic variation within the tag.

Here we address whether it is possible to capture more of the phenotypically relevant genetic diversity within a var DBL $\alpha$ tag by taking advantage of its homology block architecture. We hypothesize that since $\mathrm{HBs}$ are the 
units of sequence conservation and the means by which diversity is generated in var genes (i.e. through recombination), they may reflect functionally relevant sequence diversity that correlates with disease phenotype. To test this hypothesis, we reanalyzed the data originally analyzed by Warimwe et al. [9,10], looking for correlations between the expression of particular homology blocks and the occurrence of particular disease phenotypes. We find that a generic set of HBs, which were defined using only a few geographically distinct isolates [8], are capable of describing the variation observed at this local scale in Kenya. When we test for genotype-phenotype relationships, we find that those described by HBs are statistically stronger than those described previously. We further show that a principal component analysis (PCA) of $\mathrm{HB}$ expression rate profiles across isolates can break down $\mathrm{HB}$ variation in a way that is useful for generating high quality genotype-phenotype models.

\section{Methods}

\section{Homology block nomenclature}

The DBL $\alpha$ homology blocks discussed here are those described in Rask et al. [8]. These are distinct from the DBL $\alpha$ "homology blocks" of Smith et al. [25] and the DBL $\alpha$ "blocks" of Bull et al. [12] both in definition, and for the most part, in practice. Therefore, wherever we refer to homology blocks (HBs) below, we mean those of Rask et al., and we use their system of numbering to refer to particular HBs as well.

\section{Data and HB assessment of sequences}

The expressed sequences and the clinical data for 250 isolates (217 symptomatic, 33 asymptomatic) were obtained from the online supplementary information of [10]. The genomic sequences for 53 isolates were obtained from EMBL using the reference numbers in [9] for the genomic sequences: FN592662-FN594512. The expression rate of classic var types, which are defined by presence/absence of specific motifs in the case of cys2PoLV groups and h3sub var types, and by network analysis in the case of A-like and BS1/CP6 var types, were also obtained from the online supplementary information of [10]. All sequences were analyzed to assess $\mathrm{HB}$ composition. HBs were identified using the VarDom Server [8]. A gathering cut-off of 9.97 was used as the threshold to define a match.

\section{Linkage analysis of HBs in genomic sequences}

Linkage analysis was based on the linkage disequilibrium coefficient, D, among $\mathrm{HBs}$ within the 53 genomic isolates. The statistical significance for $\mathrm{D}$ values is determined by the method described in [26]. Where noted, D is normalized to account for the fact that $\mathrm{D}$ is maximized for intermediate frequency HBs (Additional file 1:
Figure S3). Normalization is done by dividing $\mathrm{D}$ by $(p q(1-p)(1-q))^{2}$, where $p$ and $q$ are the frequencies of the two HBs being analyzed for linkage.

\section{HB expression rate}

The $\mathrm{HB}$ expression rate for a given isolate was defined as follows: the number of HBs of a certain type found within the expressed sequences of a given isolate (the expressed sequences consist of each unique expressed sequence represented as many times as it is found within that isolate), divided by the total number of expressed sequences for that isolate.

\section{Phenotype association networks}

For the purposes of creating phenotype association networks, we analyzed the 217 symptomatic isolates within the dataset. For continuous phenotypes, we included in the network any significant correlation or rank correlation between a phenotype and an $\mathrm{HB} /$ var type expression rate or $\mathrm{PC}(\mathrm{p} \leq 0.05)$. For binary phenotypes, we included all associations where the mean expression rate or PC was found to be significantly different for the two phenotypic states ( $\mathrm{p} \leq 0.05$ by Friedman Rank, KruskalWallis and/or K-Sample T, where each test is applied only when appropriate). HBs that are linked to similar phenotypes can be defined by analyzing networks in which HBs are connected by edges to the phenotypes with which their expression is correlated. We do not correct for multiple hypothesis tests in determining these edges because the conclusions are based on the consideration of many edges taken together, and a more lenient threshold allows the network to capture a greater number of meaningful biological signals.

\section{Transformation of expression rates and rosetting level}

Prior to performing all linear and logistic regression analyses, the expression rates for particular var types (i.e., cys2, A-like, group 1, group 2, group 3, BS1/CP6 and H3sub var genes), the HB expression rates (i.e. for all 29 HBs), and the rosetting rates were transformed as described in [10]. The transformation (which is an arcsine transformation with special treatment for extreme values) is a standard method, and makes the data appropriate for fitting with regression models.

\section{Principal component analysis}

A PCA was carried out on a dataset of the HB expression rate profiles for the 217 symptomatic isolates. The expression rate profile is the set of expression rates for all $29 \mathrm{HBs}$ for a given isolate. A PCA defines differentially expressed $\mathrm{HB}$ components-i.e., orthogonal principal components (PCs). Network analyses and phenotype correlation tests were then carried out using these PCs as independent variables. To test the robustness of the 
PCA results, we repeated the PCA using non-overlapping subsets of isolates.

\section{Modeling genotype-phenotype associations}

Phenotype correlation tests consisted of multiple linear and logistic regression models, similar to the tests performed in [10], however in our case we substituted the expression rates of classic var types for $\mathrm{HB}$ expression rates, or PCs of $\mathrm{HB}$ expression rate profiles. BIC, AIC, $\mathrm{R}^{2}$ and Adjusted $\mathrm{R}^{2}$ were all used to compare the quality of alternative models. Where indicated, host age was included as an independent variable even where it did not appear to have a significant effect in order to eliminate the potential for observing spurious correlations resulting from co-correlation with this variable, since many weak correlations between disease phenotype and host age have been reported previously (e.g., [27]).

\section{Variable selection to optimize models of rosetting}

To select a set of independent variables that produce the most informative model of rosetting, we started with many possible independent variables in a multiple linear regression model, and then successively removed the least significant contributing variable, excluding host age, until the BIC stopped decreasing. We then verified that the BIC increased with the removal of any of the final independent genetic variables. The BIC, AIC, $\mathrm{R}^{2}$ and adjusted $\mathrm{R}^{2}$ scores for the final models after removing host age were also evaluated. Most variable selection procedures were also carried out under the scenario where host age is removed as soon as it is the least significant contributing variable, and in all cases examined this had no influence on the variable selection results.

\section{Identifying rosetting associated HBs or PCs}

Warimwe et al. test whether particular expression rates can significantly reduce the explanatory power of rosetting on RD as a means to identify a group of var genes that associate with rosetting and RD as opposed to impaired consciousness [10]. However, we reason that even a perfect genetic marker may not substantially reduce the effect of the rosetting coefficient. If there is a tighter relationship between rosetting and $\mathrm{RD}$ than between the expression rate of the responsible gene and RD (which is likely the case if the path from gene to rosetting to RD accumulates noise along the way), then the most informative regression model will still primarily depend on rosetting as the primary independent variable. For this reason we take a different approach. We attempt to identify rosetting-specific $v a r / \mathrm{HB}$ expression rates or PCs by considering which var/HB expression rates or PCs remain as independent predictive variables in a model of rosetting after the variable selection procedure described above.

\section{Results and discussion}

Using HBs to classify var types within a local population

Many of the HBs identified in this dataset were also found in the genome of the chimpanzee malaria parasite P. reichenowi (HBs 5, 14, 36, 64, 54, 60, 79, 210, 88, 131, $153,171,163$, and 260 , in order of frequency in the $P$. reichenowi genome). Sequence homology among such distantly related parasites reflects the ancient origin of var genes, and the strong balancing selection that maintains these sequence variants through millions of years of evolution [28].

The genomic var dataset, comprising 1851 sequences, contained 1708 unique sequences by amino acid identity (aa-types), with an average of 34.92 aa-types per isolate. There were 2-10 HBs per DBL $\alpha$ tag (Figure 1), and the genomic dataset contained 28 unique HBs in 398 unique combinations (398 HB-types), with an average of 5.19 HB-types per isolate. The cDNA dataset for all 250 isolates, comprising 4538 sequences, contained 3925 unique sequences by amino acid identity, with an average of 18.15 aa-types per isolate. These sequences contained 29 HBs in 557 unique combinations, with an average of 2.23 HB-types per isolate.

For the dataset of cDNA var tags for all 250 isolates, the average fraction of the sequence that is missed by $\mathrm{HB}$ alignment is $12.7 \%$ (when the sites before the start of the first $\mathrm{HB}$ and after the end of the final $\mathrm{HB}$ are excluded). The frequency of the HBs varied, with only a few at intermediate frequencies (Figure 2A). The sequences were highly variable in their $\mathrm{HB}$ composition (Figure 2B), and reflected the previously described recombining groups (Figure $2 \mathrm{C}$ ).

While the diversity of HB-types is almost an order of magnitude less complex than the diversity of aa-types, the former is nevertheless considerable and potentially functionally informative (Figure 3). Thus, even though these HBs were designed with reference to the var diversity of only a few parasite genomes (i.e., those analyzed in [8]), most of the sequence variation present within this local population is captured by homology to HBs, and so it is reasonable to hypothesize that the HBs capture functional variation among DBL $\alpha$ tags in this population, at least with regard to phenotypes known to be mediated by the DBL $\alpha$ domain. For example, it seems reasonable that the unique aspects of the $\mathrm{HB}$ composition observed for rosetting associated var tags (Figure 1B; Additional file 1: Figure S2) may be of functional significance.

\section{Defining groups of associated HBs through linkage or phenotype correlation networks}

With genomic samples, groups of HBs can be defined based on analyzing genomic var diversity through a simple linkage analysis of the positive linkage disequilibrium 


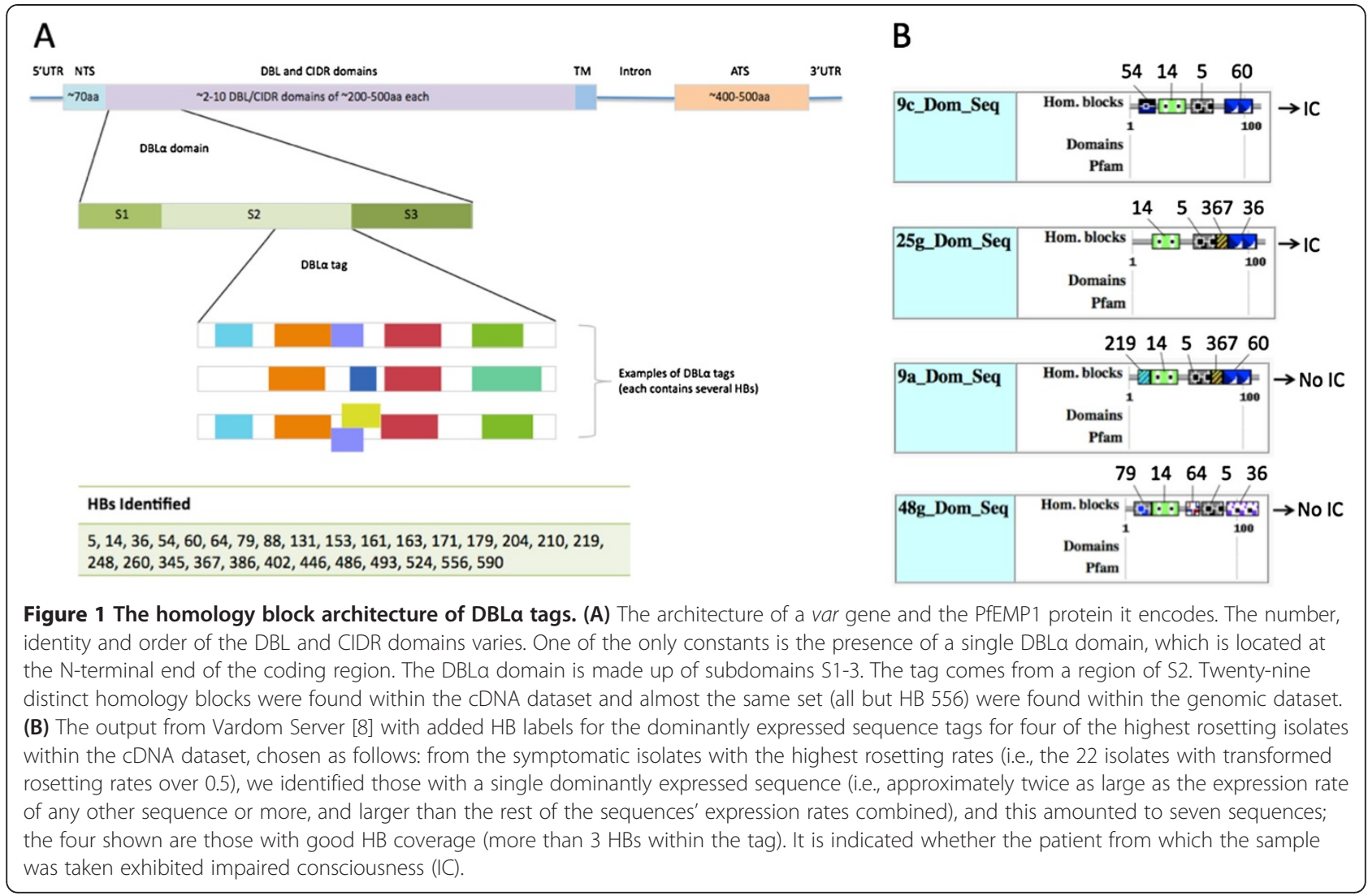

coefficient (D) values that exceed a one-tailed significance threshold of $\mathrm{p} \leq .025$ [26]. The observed number of positive pairwise linkages that lie beyond this $95 \%$ confidence interval is 65 , which greatly exceeds the expected number under the null hypothesis of random associations, 9.45. The presence of significant linkages among HBs implies that sequences are not random sets of HBs even after taking into consideration the observed HB frequencies. The weighted network of linkages among HBs (the positive normalized D values, significant and non-significant) can be analyzed for community structure (Additional file 1: Figures S3 and S4), and we find that the two communities that result from this analysis agree exactly with the two subnetworks of HBs described by the significant linkages among HBs (Figure 3A).

Using expression data, we can measure the expression rate for each $\mathrm{HB}$ in each isolate, and we observe many correlations among HB expression rates (Additional file 1: Figure S5). HB expression data also reveal that the two linkage groups of HBs are associated with very different manifestations of disease. With the observed correlations between $\mathrm{HB}$ expression rates and disease phenotypes we can build a network of significant associations between HBs and phenotypes, and define groups of HBs based on their associations with similar phenotypes. We find that two primary groups of HBs emerge from this phenotype association network (Figure $3 \mathrm{~B}$ ), and they correspond to the two groups defined by HB linkage within genomic sequences. This correspondence between the linkage and phenotype association subnetworks supports the idea that HBs may be able to serve as robust markers for functional differences among var genes.

\section{Distinguishing two subsets of A-like var tags with different phenotype correlations}

Earlier analysis of the data by Warimwe et al. established that, while A-like var expression is associated with rosetting, A-like var expression and rosetting appear to be independent with regard to their associations with disease phenotypes. Specifically, while A-like var expression is correlated with impaired consciousness but not respiratory distress, rosetting is correlated with respiratory distress but not impaired consciousness [10]. This observation led Warimwe et al. to conclude that there must be a small subset of A-like var genes that cause severe disease through a specific rosetting-dependent mechanism (Figure 4). However, their methods-which rely the expression rates of classic var types-did not reveal any statistically significant support for the existence of such a subset [10] (Additional file 2). By classic var types we henceforth mean the seven that are examined in this 


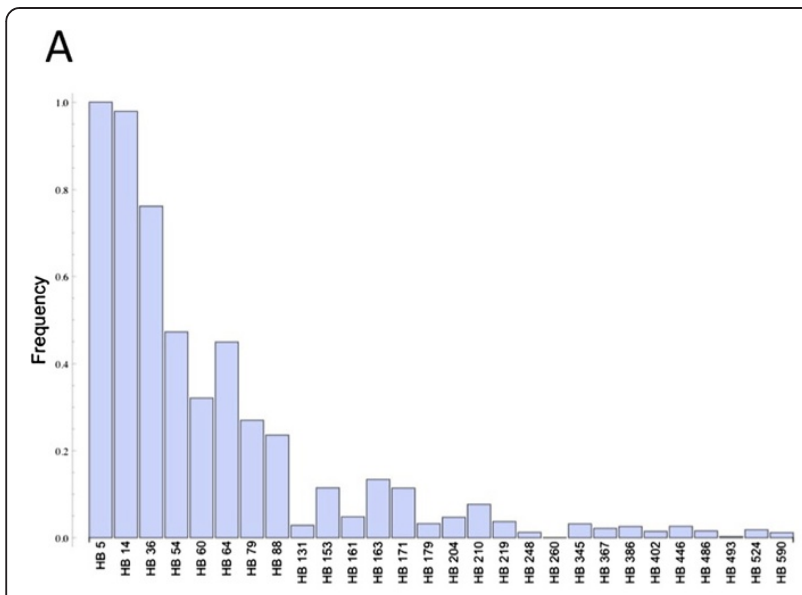

C

\section{genomic var tag sequences}

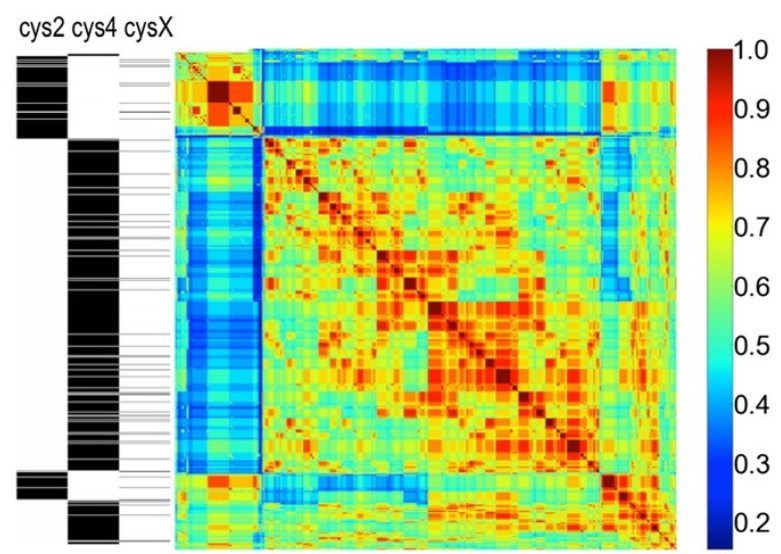

B

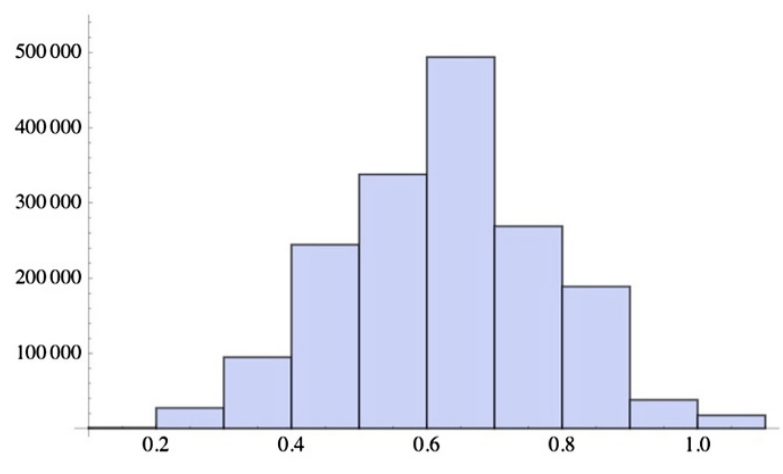

cDNA var tag sequences

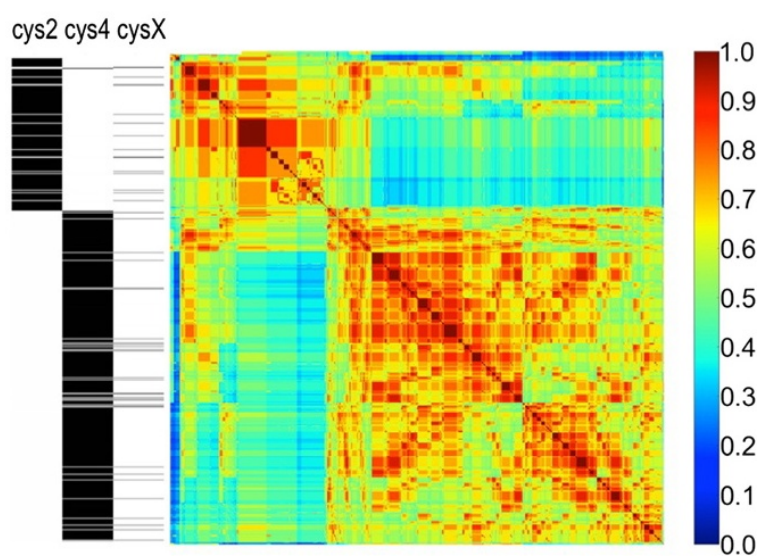

Figure 2 Var diversity within a local population is captured by homology blocks. (A) Frequency of each HB in the dataset of genomic var tags. (B-C) The pairwise similarity among sequence types, where types are defined by homology block composition: the number of HBs shared between any two sequences divided by the average number of HBs within a sequence for those two sequences. (B) Frequency distribution of pairwise HB similarities between sequences in the genomic dataset. The approximately normal distribution contrasts with the bimodal distribution that has been observed for other data, when pairwise similarity is defined by amino acid identity [29]. (C) Sequences are hierarchically ordered based on pairwise HB similarity using the average-linkage method as implemented in SciPy. The distinction between sequence tags containing two cysteines (cys2) versus four (cys4) is very clear, reflecting that recombination occurs at a faster rate within, relative to between, the two groups.

prior analysis: cys2, A-like, the H3 subset (h3sub), cysPoLV groups 1, 2, and 3, and BS1/CP6 [10].

In an attempt to identify this hypothesized class of var genes using HBs, we looked for a subset of A-like var genes that have expression rates significantly correlated with rosetting, and simultaneously significantly anticorrelated with IC. Among the expression rates of classic var types, none had significant and opposite associations with rosetting and $\mathrm{IC}$. Among the $\mathrm{HB}$ expression rates we tested, there were many with significant associations with rosetting (data not shown, but see Additional file 1: Figure S9 ) and/or IC (Additional file 1: Figure S8), but only one had significant associations with these phenotypes in opposite directions: The expression rate of
HB 204 is significantly anti-correlated with rosetting $(\mathrm{p}=0.025)$ and significantly correlated with IC ( $\mathrm{p}=$ 0.0069 ) in models using HB 204 and host age as the only independent variables (Additional file 1: Figure S8).

Next we addressed whether any HBs can provide additional information about rosetting, beyond what is already captured by classic var tag typing methods. We added each $\mathrm{HB}$ expression rate as an additional independent variable, one at a time, into a model of rosetting that already contained eight other independent variables: host age and the expression rates for the classic var types. We then compared model statistics (primarily $\mathrm{BIC}$, but also $\mathrm{AIC}, \mathrm{R}^{2}$ and adjusted $\mathrm{R}^{2}$ ) to determine the benefit of the particular HB expression rate to the model 


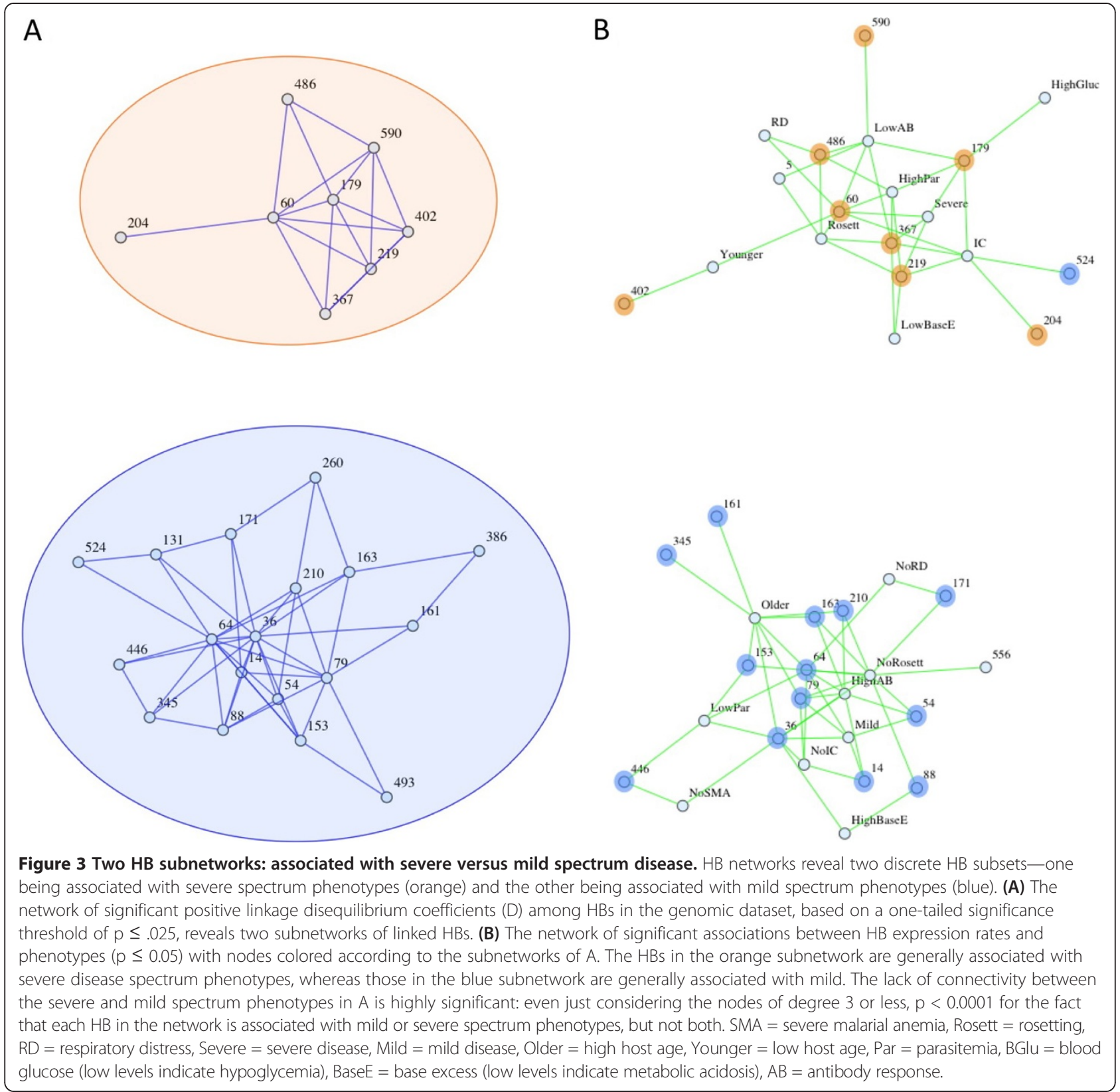

(Additional file 3: Table S1). While most HBs increase the $\mathrm{BIC}$, decrease the adjusted $\mathrm{R}^{2}$ and provide an insignificant contribution to predicting rosetting ( $p>>0.05)$, two HBs make improvements to the model and have significant p-values even within these over-parameterized models. HB 204 substantially reduces the BIC (from 50.72 down to 48.62 ), and substantially increases the adjusted $R^{2}$ (from 0.348 up to 0.376 ). HB 54 is the only other HB to reduce the $\mathrm{BIC}$ and increase the adjusted $\mathrm{R}^{2}$ of the original model, however it only brings the BIC down slightly (to 50.65) and the adjusted $\mathrm{R}^{2}$ up slightly (to 0.367 ) (Additional file 3: Table S1).

\section{Variable selection to achieve a model of rosetting}

In order to identify what genetic variation best explains the variation observed in rosetting, we performed a variable selection procedure to find the optimal set of independent variables for a multiple regression model of rosetting. Three tests were performed, which together show that HB 219 is a better predictor of rosetting than any of the classic var types (Table 1):

In a first test, we start with a model that initially includes all seven classic var types plus host age. We successively remove the genetic variable that contributes least significantly to the model until the BIC and related 


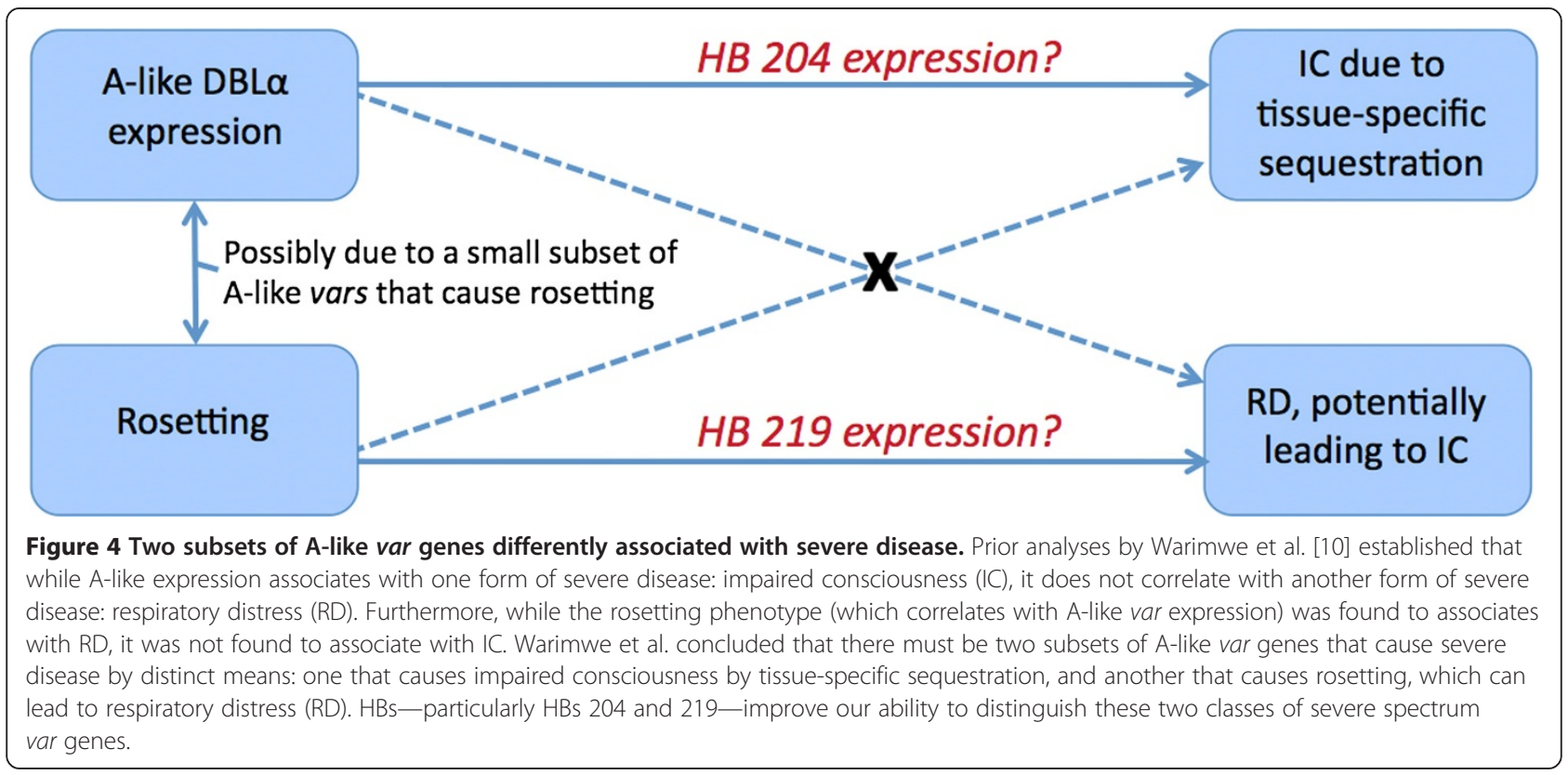

statistics are optimized (see Methods for details). We find that the model with the lowest BIC contains the expression rates for cys 2 and BS1/CP6 var types as positive predictors of rosetting, and the expression rates for cysPoLV group 2 and cysPoLV group 3 var types as negative predictors of rosetting $(\mathrm{BIC}=37.40)$ (row A in Table 1 and Additional file 3: Table S3).

In a second test we start with all $29 \mathrm{HB}$ expression rates plus host age as independent variables and then we follow the same variable selection procedure. In this case the resulting model is one with $\mathrm{HB} 36, \mathrm{HB} 204$ and $\mathrm{HB}$ 210 as negative predictors of rosetting, and HB 219 and $\mathrm{HB} 486$ as positive predictors of rosetting $(\mathrm{BIC}=36.60)$ (row B in Table 1 and Additional file 3: Table S3).

In a third variable selection test we start with all 29 $\mathrm{HB}$ expression rates in addition to the expression rates for all seven classic var types, plus host age. Starting with this initial set of independent variables, the model that results after variable selection is one containing the

Table 1 Statistics for multiple regression models predicting rosetting*

\begin{tabular}{llllll}
\hline \multicolumn{1}{l}{ Independent variables } & AIC & BIC & R2 & Adj. R2 \\
\hline A & Cys2, Grp2, Grp3, BS1CP6 & 20.14 & 37.40 & 0.358 & 0.338 \\
B & HB36, HB204, HB210, HB219, HB486 & 16.48 & 36.60 & 0.385 & 0.361 \\
C & BS1CP6, HB54, HB171, HB204, HB219 & 14.02 & 34.14 & 0.400 & 0.373 \\
D & BS1CP6, PC1, PC3, PC4, PC22 & 4.776 & 24.90 & 0.438 & 0.415 \\
\hline
\end{tabular}

*The result of removing the least significant genetic variable, one by one, from models of rosetting that start with the expression rates of: (row A) the 7 classic var types, (row B) the $29 \mathrm{HB}$ expression rates, (row C) the expression rates for both the 7 classic var types and the $29 \mathrm{HBs}$, and (row D) the expression rates for the 7 classic var types and the 29 PCs. The variable selection procedure is done maintaining host age in the model, however statistics are shown with age removed. Positive effect independent variables are shown in boldface. expression rates of BS1/CP6 and HB 219 as positive predictors of rosetting, and the expression rates of $\mathrm{HB} 54$, HB 171 and HB 204 as negative predictors of rosetting $(\mathrm{BIC}=34.14)$ (row $\mathrm{C}$ in Table 1 and Additional file 3: Table S3; Additional file 1: Figure S10).

Two additional anecdotes provide further credibility to our finding that HB 219 expression rate is a robust positive predictor of rosetting: First, we find that in all of the nine cases where there is rosettting data for an isolate that has $\mathrm{HB} 219$ present in its most highly expressed sequence, considerable rosetting is observed (defined as $>0.1$ ). Secondly, we find that the DBL $\alpha$ domains of known rosetting var genes [30,31] contain HB 219 (Additional file 1: Figure S2).

Based on a comparison of the BIC scores of the models that result from the above variable selection procedures (Table 1), it seems that a more informative model for rosetting can be achieved when $\mathrm{HB}$ expression rates are used as candidate independent variables in addition to classic var types. More specifically, the most informative model is achieved when we consider the expression rates of several $\mathrm{HBs}$ in addition to the expression rates of one classic var type: BS1/CP6. This becomes even clearer when we perform a fourth variable selection procedure using the principal components discussed below (row D in Table 1 and Additional file 3: Table S3).

\section{Principal components of $\mathrm{HB}$ expression rate profiles and variation in rosetting}

We perform a PCA on the HB expression rate profile, which we define as the set of expression rates for all 29 HBs. This deconstructs the $\mathrm{HB}$ expression rate profiles into orthogonal principal components (PCs) based on 
how they vary across different isolates. We then repeat the above network and variable selection analyses using $\mathrm{PCs}$ in place of individual $\mathrm{HB}$ expression rates (Additional file 1: Figures S11 and S12).

We find that PC 1 is related to the cys 2 versus noncys 2 distinction (Figure 5B), and that it captures the difference between $\mathrm{HBs}$ that are associated with severe versus mild spectrum phenotypes (Figure 3; Additional file 1: Figure S4). PC 1 correlates with all of the severe spectrum phenotypes (Figure $5 \mathrm{E}$ ) and the $\mathrm{HB}$ expression rates that contribute most to $\mathrm{PC} 1$ are those with strong associations with disease phenotypes. PC 1 describes $8.15 \%$ of the variation among isolates with regard to their HB expression rates (Additional file 1: Figure S14). The HBs that have large positive values in PC 1 define the core of the mild spectrum linkage/phenotype subnetwork (Figures 3, 5A and D; Additional file 1: Figures S4 and S13). Likewise, the $\mathrm{HB}$ that has the dominant negative value in $\mathrm{PC} 1, \mathrm{HB} 60$, defines the core of the severe spectrum linkage/phenotype subnetwork (Figures 3, 5A and C; Additional file 1: Figures S4 and S13). These observations about PC 1 are robust to the specific isolates used for the PCA. When non-overlapping subsets of isolates are analyzed separately, the relative contributions of the various $\mathrm{HB}$ expression rates that primarily contribute to PC 1 remain essentially the same (Additional file 1: Figure S15).

We address whether the PCs provide additional information about rosetting beyond what can be predicted based on the expression rates of the classic var types. We start with a multiple regression model of rosetting that has the seven classic var types, plus host age, as independent variables. We then add each of the PCs, one at a time, and observe whether they make a significant contribution to predicting rosetting and/or reduce the $\mathrm{BIC}$ of the model. The only PC that is significantly predictive about rosetting in the context of this already over-parameterized model is PC 3 , which shows a positive association with rosetting. PC 3 is also the only PC to reduce the BIC (from 50.72 down to 48.36), and it also reduces the AIC (from 21.97 down to 16.73) and increases the adjusted $\mathrm{R}^{2}$ (from 0.348 to 0.378 ) (Additional file 3: Table S2).

The above findings suggest that, regarding the rosetting pattern, PC 3 provides qualitatively different information from any of the classic var types. PC 3 is dominated by a strong negative value in the dimension of $\mathrm{HB} 204$ expression rate (Figure 5A), which is consistent with PC 3 having a positive association with rosetting, since we established above that HB 204 significantly anti-correlates with rosetting.

Next we perform a variable selection procedure to address whether an optimized model of rosetting will contain PCs or classic var types, or both. We start with a multiple regression model of rosetting that includes all 29 PCs and all seven classic var types, and host age, as the independent variables. We follow the variable selection procedure (described in Methods), and we find that the most informative model by $\mathrm{BIC}$ includes the following genetic variables: BS1/CP6, PC 1, PC 3, PC 4 and PC 22 (row D in Table 1 and Additional file 3: Table S3) $(\mathrm{BIC}=24.90)$.

The PC-containing models have much lower BIC scores and higher adjusted $\mathrm{R}^{2}$ values compared to all other models (row D in Table 1 and Additional file 3: Table S3). This means that the PCA is able to consolidate the relevant functional variation into fewer variables by replacing a handful of $\mathrm{HB}$ expression rates with a single $\mathrm{PC}$ and still retaining the same ability to predict rosetting. For example, relative to any individual expression rate, PC 1 appears to be a better predictor of whether an isolate will express severe spectrum phenotypes or mild spectrum phenotypes. Thus, the expression rates of many HBs appear to be non-independent with respect to their relationships to phenotype. Our PCA results also imply that within the small DBL $\alpha$ tag there are multiple independent genetic components that are relevant to disease phenotype, since otherwise we would not expect to find more than one $\mathrm{PC}$ playing a significant role in any of the phenotype prediction models. This conclusion is consistent with the fact that many of the first several PCs explain similar levels of variation among isolates (Additional file 1: Figure S13 and S14).

The principal components improve phenotype prediction, but they are less straightforward to interpret than individual $\mathrm{HB}$ expression rates. Nevertheless, our results demonstrate that PC 1 clearly corresponds to the major division found by network analyses, severe and mild spectrum associated var genes.

Furthermore, the various correlations between phenotypes and PCs, and between the expression rate of various sequence types and PCs, can be summarized in networks, which can provide additional means to interpret the PCs (Figure 5E; Additional file 1: Figure S11). In summary, we find that two PCs capture interesting phenotypic distinctions among isolates, and we find that model BICs improve considerably when PCs are used in place of individual $\mathrm{HB}$ expression rates.

\section{The consistency of HB-phenotype associations in distinct populations}

HB analysis of a smaller dataset from Mali that was originally analyzed by Kyriacou et al. [14], reveals that at least some of the HB-phenotype associations reported above are similarly informative in geographically distinct (and presumably genetically unrelated) populations. Twenty-four of the $29 \mathrm{HBs}$ we identified in the Kenyan dataset (Figure 1) were present in the Malain dataset 


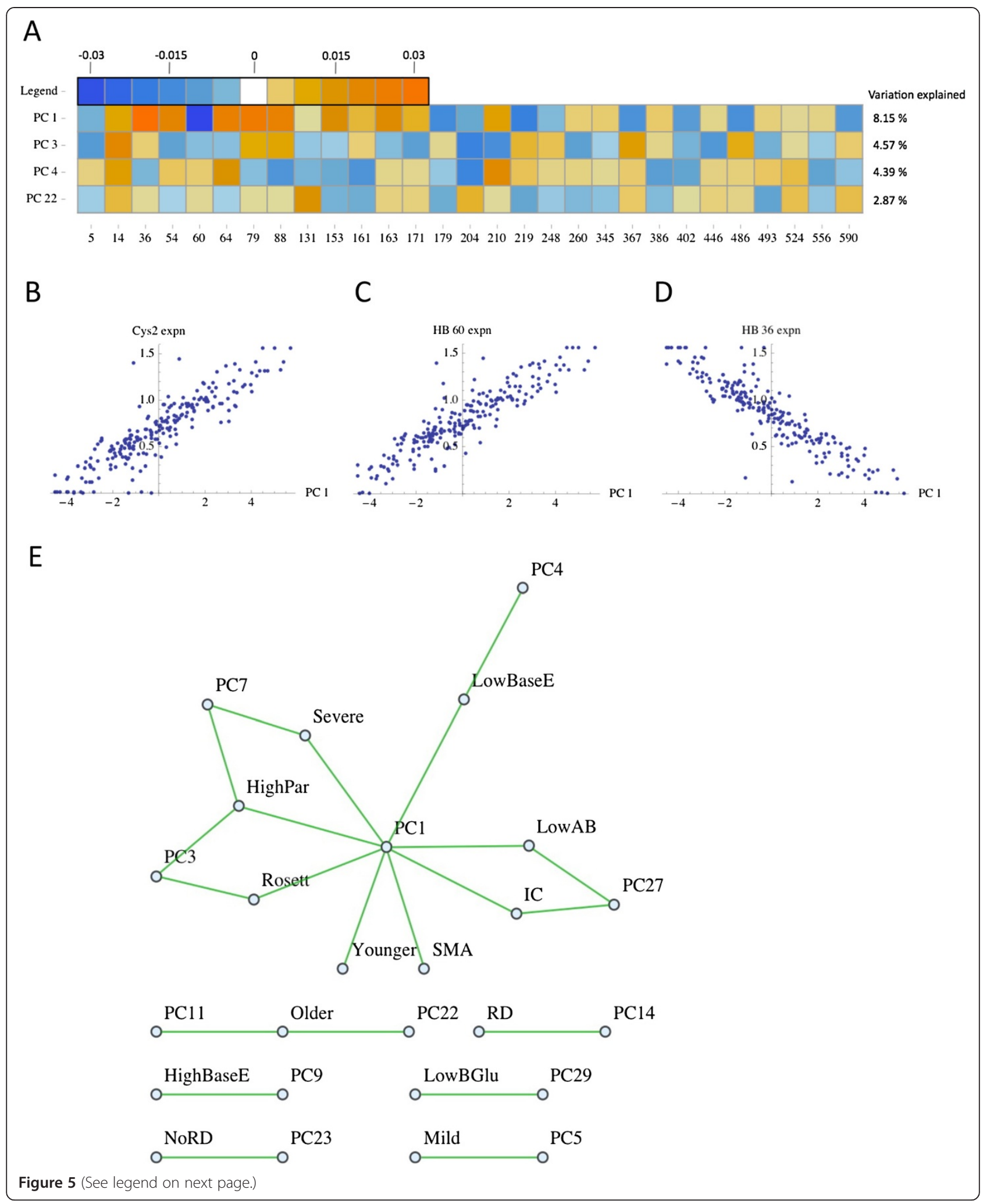


(See figure on previous page.)

Figure 5 Principal components of HB expression rate profiles. (A) PCs expressed as coordinates in the original space of the data, which is the expression rates for all $29 \mathrm{HBs}$ for each of the isolates. The amount of variation among the isolates that is explained by each of the PCs is shown on the right. (B) The PCA of HB expression rate profiles reflects the differentially expressed HB components, and the first PC defines the extent to which there is a bias toward the expression of var tags with 2 cysteines (cys2). The cys2 expression bias maps roughly to an association with mild versus severe disease spectrum phenotypes. (C) PC1 (and Cys2 var gene expression) correlates with the expression of several HBs, including HB 60. (D) PC1 (and Cys2 var gene expression) anti-correlates with the expression of several HBs, including HB 36. (E) The network of significant correlations between $\mathrm{HB}$ expression rate profile principal components (PCs) and disease phenotypes $(p \leq 0.05)$. SMA = severe malarial anemia, Rosett = rosetting, $\mathrm{RD}=$ respiratory distress, Severe = severe disease, Mild = mild disease, Older $=$ high host age, Younger = low host age, Par $=$ parasitemia,

BGlu = blood glucose (low levels indicate hypoglycemia), BaseE = base excess (low levels indicate metabolic acidosis), AB = antibody response.

(data not shown). The Malian dataset contains 9 isolates from cerebral cases of malaria, and 8 isolates that serve as negative control for severe disease since they are from mild hyperparasitemic cases. Kyriacou et al. argue that mild hyperparasitemic malaria is the appropriate negative control for cerebral malaria since the two forms of disease exhibit comparable levels of parasitemia. Given the null hypothesis that the probability of expressing a certain var type is the same in both the mild hyperparasitaemic malaria patients and cerebral malaria patients, we calculate the probability (by Fisher's exact test) of the data observed by Kyriacou et al., and we find that the distribution of HB 36 is less likely than the distribution of cys 2 -indicating that $\mathrm{HB} 36$ is a stronger marker of severe disease than cys 2 in the Malian population. This is essentially what we observed in the Kenyan population, since $\mathrm{HB} 36$ is the dominant $\mathrm{HB}$ expression rate of the $\mathrm{PC}$ that correlates most strongly with severe disease, PC 1 (Figure 5E). Additionally, in the Malian population we find that HBs 60, 64, 79, 163, and 179 are differentially expressed in cerebral versus mild hyperparasitaemic cases $(\mathrm{p}<.05)$.

For the Malian dataset [14], we also compare the recall (hit rate), accuracy and precision of the following two predictive models: (1) expressed DBL $\alpha$ sequence tags containing two cysteines predict severe malaria whereas those with some other number predict mild hyperparasitaemic malaria, and (2) expressed sequence tags lacking HB 36 predict severe malaria whereas those with HB 36 predict mild disease. The hit rate, accuracy and precision are given by $\mathrm{TP} / \mathrm{P},(\mathrm{TP}+\mathrm{TN}) /(\mathrm{P}+\mathrm{N})$ and $\mathrm{TP} /(\mathrm{TP}+\mathrm{FP})$, respectively, where TP is the number of truly positive instances classified as positive, $\mathrm{TN}$ is the number of truly negative instances classified as negative, FP is the number of truly negative instances classified as positive, $\mathrm{P}$ is the total number of truly positive instances classified as either positive or negative, and $\mathrm{N}$ is the total number of truly negative instances classified as either positive or negative [32]. For the purpose of predicting severe disease from sequence features of expressed DBL $\alpha$ var tags in the Malian population, classification by HB 36 outperforms classification by cys 2 in terms of all three of the above. The hit rate is 0.723 as opposed to 0.617 , the accuracy is 0.765 as opposed to 0.724 , and the precision is 0.773 as opposed to 0.763 .

Among the unique set of sequences expressed within the cerebral and hyperparasitemia isolates, the rank correlations (both Spearman and Kendall) of rosetting with each of HB 60, 79, 153, and 219 are all greater in magnitude than the rank correlation of rosetting with cys2. These several HBs are also associated with rosetting in the Kenyan dataset [10], and thus, they appear to serve as more informative predictors of rosetting than the number of cysteines within the var DBL $\alpha$ tag.

\section{Conclusions}

Even though the HBs were designed using a very small number of var sequences isolated from a few parasite genomes, they manage to cover the sequence diversity of a local population, leaving only the minority of sites unaligned. We find that the variation described by $\mathrm{HB}$ diversity within the var DBL $\alpha$ tag is not completely redundant with the diversity already described by classic methods. Furthermore, relative to classic methods, the consideration of HB composition appears to be more informative for predicting whether a tag's expression is associated with various disease phenotypes.

All of the HBs within the optimized rosetting model (HBs 171, 204, 54 and 219; row C in Table 1) are located at the N-terminal end of the tag (Additional file 1: Figure S16). They are also overlapping with the PoLV1 site (position 3-5 in each of the above HBs), which distinguishes cysPoLV group 1 var genes from other cys2 var genes. Based on the defining HMM for HB 204 (Additional file 1: Figure S16) and the definition of cysPoLV group 1, it is clear that HB 204 expression should anti-correlate with cysPoLV group 1 expression, and indeed it does (Additional file 1: Figure S17). From the network analyses (Figure 3; Additional file 1: Figure S4) it can be seen that HB 54 and $\mathrm{HB} 171$ are in the mild spectrum subnetwork, and HB 219 and HB 204 are in the severe spectrum subnetwork. Therefore, HB 204 is unusual in that it maps to the severe spectrum subnetwork, but nevertheless anti-correlates with rosetting. No other $\mathrm{HB}$ or classic var type shows this pattern, reflecting the fact HB 204 contains unique information that is potentially 
useful for refining our understanding of the different mechanisms underlying severe disease.

HB 204 expression rate is a significant negative predictor of rosetting regardless of the details of the model. However, its expression is positively correlated with the expression of cysPoLV group 2 tags (correlation coefficient $\left.=0.434, \mathrm{p}<10^{-10}\right)$, which are by definition cys2. CysPoLV group 2 var expression does not predict rosetting in this dataset, either positive or negatively-so possibly HB 204 marks a subset of group 2 var genes that do not cause rosetting but that nevertheless cause severe disease, since HB 204 expression is significantly associated with impaired consciousness (however, it is worth noting that HB 204 is also found in var genes other than cysPoLV group 2). A final interesting anecdote about HB 204 is that it is part of domain cassette 17 of IT4var13, which is one of the sequence variants known to mediate binding to brain endothelial cells [21].

Warimwe et al. put forward the hypothesis that there are at least two classes of A-like var genes: those that cause rosetting and that can lead to RD in severe cases, and those that cause impaired consciousness through a tissue-specific mechanism that does not rely on rosetting (Figure 4) [10]. HB 204 may therefore serve as an ideal marker to distinguish between these two types of severe spectrum genes. Its absence, particularly in the cys2 context, indicates the rosetting phenotype. Its presence marks low rosetting var genes that are nevertheless associated with severe disease by way of impaired consciousness.

HB 219 is also interesting because, while its expression is correlated with cysPoLV group 1 expression (Additional file 1: Figures S16 and S17), its expression is more tightly associated with rosetting than cysPoLV group 1 expression is. This is evident in the coefficients and statistical significance of the regression models for rosetting (data not shown), and in the fact that HB 219 expression is an independent variable within the most informative models for rosetting while cysPoLV group 1 expression falls out (Table 1). Since the sequence covered by HB 219 is considerably longer than the MFK motif that defines cysPoLV group 1 var genes, it is likely that HB 219 covers additional sequence variation that is either directly or indirectly linked to the rosetting phenotype. Furthermore, HB 219 expression correlates with both high parasitemia and hypoglycemia (Figure 3B). Both of these associations further support the hypothesis that HB 219 is linked to a form of severe disease that manifests through overall high parasite burden rather than through tissue-specific sequestration.

Within the Kenyan population that is the focus of this study, HB expression rates (and to an even greater extent, PCs of $\mathrm{HB}$ expression rate profiles) improve our ability to differentiate mild versus severe spectrum var genes beyond what is possible with classic typing methods. Furthermore, HBs appear to be informative markers of disease phenotype in more than just this particular population. In a dataset from Mali we again find that HB 219 expression is significantly associated with high levels of rosetting, and that the $\mathrm{HB}$ composition of the expressed var sequence tags-particularly with respect to HB 36predicts disease severity with higher precision, accuracy and recall than classic methods. These results suggest that the DBL $\alpha$ HB-phenotype associations, which we characterized using the large Kenyan dataset, are consistent across distinct populations. Thus, a single set of DBL $\alpha$ HBs can potentially serve as parasite genetic markers for severe disease phenotypes in geographically diverse populations. Moreover, the fact that many of the same HB-phenotype relationships are found in two geographically distant populations supports the idea that there is a functional link between particular DBL $\alpha$ HBs and the molecular mechanisms underlying severe disease, since otherwise we would expect recombination to alter HB-phenotype linkages.

In summary, HB typing methods allow for the construction of more specific genotype-phenotype models that in turn suggest that two distinct molecular mechanisms underlie severe malaria. Specifically, we find that var DBL $\alpha$ HB 204 expression predicts a form of severe disease that is associated with impaired consciousness and the absence of rosetting, and that var DBL $\alpha$ HB 219 expression predicts a form of severe disease that is associated with high rosetting. Insights into genotypephenotype associations within this system can potentially aid in the development of new diagnostic and monitoring tools for malaria, and perhaps even future vaccines, since var genes have been implicated as possible future vaccine targets [33]. Furthermore, if additional studies are undertaken that assess both var expression and clinical symptoms, it should be possible to further refine our descriptions of these genotype-phenotype relationships.

Lastly, HBs have the potential to elucidate complex ecological and evolutionary dynamics that potentially shape antigenic diversity within $P$. falciparum populations (e.g., [34]). For example, the fact that the same conserved set of HBs can describe var sequence diversity at multiple geographic scales and locations reveals strong balancing selection to maintain ancient sequence fragments across vast expanses of time and space. The complex ecological and evolutionary dynamics that are at play warrant further study because they likely shape $P$. falciparum antigenic diversity, and in so doing, strongly impact the epidemiology of malaria.

\section{Additional files}

Additional file 1: Additional figures. Figure S1. Respiratory distress

(RD) as a function of host age and rosetting. Figure S2. HB composition of known rosetting var genes. Figure S3. Linkage disequilibrium coefficient (D) values for all pairs of HBs in the genomic dataset. Figure S4. 
Community partition of weighted linkage network of HBs. Figure S5. HB-HB expression rate correlation matrix. Figure S6. Model of respiratory distress. Figure S7. Relationship between rosetting and respiratory distress. Figure S8. Relationship between impaired consciousness and the expression of various var types and HBs. Figure S9. The best fit relationship between six variables and rosetting using a window analysis. Figure S10. Relationship between rosetting and expression rates of var types and HBs. Figure S11. PC-classic var type association network. Figure S12. PC-HB relationships. Figure S13. Principal components in data space. Figure S14. The amount of variation explained by each PC. Figure S15. PCA for two subsets of the data. Figure S16. Representation of select homology blocks. Figure S17. HB-classic var type association network.

\section{Additional file 2: Further explanation of methods.}

\section{Additional file 3: Additional tables. Table S1. Multiple regression} models of rosetting that include an HB expression rate as an independent variable. Table S2. Multiple regression models of rosetting that include an HB expression PC as an independent variable. Table S3. Statistics for multiple regression models predicting rosetting with and without age.

\section{Competing interests}

The authors declare no competing interests.

\section{Authors' contributions}

MMR conceived of the study, carried out the analysis and wrote the manuscript. KPD, MP and TSR contributed to the study design and critically revised the manuscript. EBB contributed to the data analysis and critically revised the manuscript. All authors read and approved the final manuscript.

\section{Acknowledgements}

We thank Donald S. Chen and Yael Artzy-Randrup for helpful input related to this work. MP is an Investigator at Howard Hughes Medical Institute. EBB was supported by a Department of Energy Computational Science Graduate Fellowship (grant DE-FG02-97ER25308).

\section{Author details}

'Department of Ecology and Evolutionary Biology, University of Michigan, 2019 Kraus Nat. Sci. Bldg., 830 North University Ave, Ann Arbor 48109-1048, Michigan, USA. ${ }^{2}$ Howard Hughes Medical Institute, Ann Arbor, Michigan, USA. ${ }^{3}$ Department of Microbiology, Division of Medical Parasitology, New York University School of Medicine, New York City, New York, USA. ${ }^{4}$ Center for Computational Medicine and Bioinformatics, University of Michigan, Ann Arbor, Michigan, USA.

Received: 18 July 2013 Accepted: 26 October 2013 Published: 6 November 2013

\section{References}

1. Chan JA, Howell KB, Reiling L, Ataide R, Mackintosh CL, Fowkes FJ, Petter M, Chesson JM, Langer C, Warimwe GM, et al: Targets of antibodies against plasmodium falciparum-infected erythrocytes in malaria immunity. J Clin Invest 2012, 122(9):3227-3238.

2. Chen DS, Barry AE, Leliwa-Sytek A, Smith TA, Peterson I, Brown SM, Migot-Nabias F, Deloron P, Kortok MM, Marsh K, et al: A molecular epidemiological study of var gene diversity to characterize the reservoir of plasmodium falciparum in humans in Africa. PLoS One 2011, 6(2):e16629.

3. Kraemer SM, Smith JD: A family affair: var genes, PfEMP1 binding, and malaria disease. Curr Opin Microbiol 2006, 9(4):374-380.

4. Freitas-Junior LH, Bottius E, Pirrit LA, Deitsch KW, Scheidig C, Guinet F, Nehrbass $U$, Wellems TE, Scherf A: Frequent ectopic recombination of virulence factor genes in telomeric chromosome clusters of P. falciparum. Nature 2000, 407(6807):1018-1022.

5. Taylor HM, Kyes SA, Newbold Cl: Var gene diversity in plasmodium falciparum is generated by frequent recombination events. Mol Biochem Parasitol 2000, 110(2):391-397.

6. Bopp SE, Manary MJ, Bright AT, Johnston GL, Dharia NV, Luna FL, McCormack S, Plouffe D, McNamara CW, Walker JR, Fidock DA, Denchi EL, Winzeler EA: Mitotic evolution of Plasmodium falciparum shows a stable core genome but recombination in antigenic gene families. PLOS Genetics 2013, 9(2):e1003293.

7. Frank M, Kirkman L, Costantini D, Sanyal S, Lavazec C, Templeton TJ, Deitsch KW: Frequent recombination events generate diversity within the multi-copy variant antigen gene families of plasmodium falciparum. Int J Parasitol 2008, 38(10):1099-1109.

8. Rask TS, Hansen DA, Theander TG, Gorm Pedersen A, Lavstsen T: Plasmodium falciparum erythrocyte membrane protein 1 diversity in seven genomes-divide and conquer. PLoS Comput Biol 2010, 6(9):e1000933.

9. Warimwe GM, Keane TM, Fegan G, Musyoki JN, Newton CR, Pain A, Berriman M, Marsh K, Bull PC: Plasmodium falciparum var gene expression is modified by host immunity. Proc Natl Acad Sci USA 2009, 106(51):21801-21806.

10. Warimwe GM, Fegan G, Musyoki JN, Newton CR, Opiyo M, Githinji G, Andisi C, Menza F, Kitsao B, Marsh K, et al: Prognostic indicators of lifethreatening malaria are associated with distinct parasite variant antigen profiles. Sci Transl Med 2012, 4(129):129ra145.

11. Bull PC, Kyes S, Buckee CO, Montgomery J, Kortok MM, Newbold Cl, Marsh $\mathrm{K}$ : An approach to classifying sequence tags sampled from plasmodium falciparum var genes. Mol Biochem Parasitol 2007, 154(1):98-102.

12. Bull PC, Buckee CO, Kyes S, Kortok MM, Thathy V, Guyah B, Stoute JA, Newbold Cl, Marsh K: Plasmodium falciparum antigenic variation: mapping mosaic var gene sequences onto a network of shared, highly polymorphic sequence blocks. Mol Microbiol 2008, 68(6):1519-1534

13. Normark J, Nilsson D, Ribacke U, Winter G, Moll K, Wheelock CE, Bayarugaba J, Kironde F, Egwang TG, Chen Q, et al: PfEMP1-DBL1alpha Amino acid motifs in severe disease states of plasmodium falciparum malaria. Proc Natl Acad Sci USA 2007, 104(40):15835-15840.

14. Kyriacou HM, Stone GN, Challis RJ, Raza A, Lyke KE, Thera MA, Kone AK, Doumbo OK, Plowe CV, Rowe JA: Differential var gene transcription in Plasmodium falciparum isolates from patients with cerebral malaria compared to hyperparasitaemia. Mol Biochem Parasit 2006 150(2):211-218

15. Bull PC, Berriman M, Kyes S, Quail MA, Hall N, Kortok MM, Marsh K, Newbold Cl: Plasmodium falciparum variant surface antigen expression patterns during malaria. PLoS pathogens 2005, 1(3):e26.

16. Newbold C, Warn P, Black G, Berendt A, Craig A, Snow B, Msobo M, Peshu N, Marsh K: Receptor-specific adhesion and clinical disease in plasmodium falciparum. Am J Trop Med Hyg 1997, 57(4):389-398.

17. Heddini A, Pettersson F, Kai O, Shafi J, Obiero J, Chen Q, Barragan A, Wahlgren M, Marsh K: Fresh isolates from children with severe plasmodium falciparum malaria bind to multiple receptors. Infect Immun 2001, 69(9):5849-5856.

18. Rowe JA, Moulds JM, Newbold Cl, Miller LH: P. falciparum rosetting mediated by a parasite-variant erythrocyte membrane protein and complement-receptor 1. Nature 1997, 388(6639):292-295.

19. Carlson J, Helmby H, Hill AV, Brewster D, Greenwood BM, Wahlgren M: Human cerebral malaria: association with erythrocyte rosetting and lack of anti-rosetting antibodies. Lancet 1990, 336(8729):1457-1460.

20. Juillerat A, Lewit-Bentley A, Guillotte M, Gangnard S, Hessel A, Baron B, Vigan-Womas I, England P, Mercereau-Puijalon O, Bentley GA: Structure of a plasmodium falciparum PfEMP1 rosetting domain reveals a role for the $\mathrm{N}$-terminal segment in heparin-mediated rosette inhibition. Proc Natl Acad Sci USA 2011, 108(13):5243-5248.

21. Avril M, Tripathi AK, Brazier AJ, Andisi C, Janes JH, Soma VL, Sullivan DJ Jr, Bull PC, Stins MF, Smith JD: A restricted subset of var genes mediates adherence of plasmodium falciparum-infected erythrocytes to brain endothelial cells. Proc Natl Acad Sci USA 2012, 109(26):E1782-E1790.

22. Bertin Gl, Lavstsen T, Guillonneau F, Doritchamou J, Wang CW, Jespersen JS, Ezimegnon S, Fievet N, Alao MJ, Lalya F, et al: Expression of the domain cassette 8 plasmodium falciparum erythrocyte membrane protein 1 is associated with cerebral malaria in Benin. PLOS One 2013, 8(7):e68368.

23. Lavstsen T, Turner L, Saguti F, Magistrado P, Rask TS, Jespersen JS, Wang CW, Berger SS, Baraka V, Marquard AM, et al: Plasmodium falciparum erythrocyte membrane protein 1 domain cassettes 8 and 13 are associated with severe malaria in children. Proc Natl Acad Sci USA 2012, 109(26):E1791-E1800.

24. Claessens A, Adams Y, Ghumra A, Lindergard G, Buchan CC, Andisi C, Bull PC, Mok S, Gupta AP, Wang CW, et al: A subset of group A-like var genes 
encodes the malaria parasite ligands for binding to human brain endothelial cells. Proc Natl Acad Sci USA 2012, 109(26):E1772-E1781.

25. Smith JD, Subramanian G, Gamain B, Baruch DI, Miller LH: Classification of adhesive domains in the plasmodium falciparum erythrocyte membrane protein 1 family. Mol Biochem Parasitol 2000, 110(2):293-310.

26. Hedrick P, Jain S, Holden L: Multilocus systems in evolution. Evol Biol 1978, 11:101-184.

27. Roca-Feltrer A, Carneiro I, Smith L, Schellenberg JR, Greenwood B, Schellenberg D: The age patterns of severe malaria syndromes in sub-Saharan Africa across a range of transmission intensities and seasonality settings. Malar J 2010, 9:282.

28. Zilversmit MM, Chase EK, Chen DS, Awadalla P, Day KP, McVean G: Hypervariable antigen genes in malaria have ancient roots. BMC Evol Biol 2013, 13:110.

29. Barry AE, Leliwa-Sytek A, Tavul L, Imrie H, Migot-Nabias F, Brown SM, McVean GA, Day KP: Population genomics of the immune evasion (var) genes of plasmodium falciparum. PLoS pathogens 2007, 3(3):e34

30. Angeletti D, Albrecht L, Blomqvist K, Quintana Mdel P, Akhter T, Bachle SM, Sawyer A, Sandalova T, Achour A, Wahlgren M, et al: Plasmodium falciparum rosetting epitopes converge in the SD3-loop of PfEMP1-DBL1alpha. PLoS One 2012, 7(12):e50758.

31. Albrecht L, Moll K, Blomqvist K, Normark J, Chen Q, Wahlgren M: var gene transcription and PfEMP1 expression in the rosetting and cytoadhesive plasmodium falciparum clone FCR3S1.2. Malar J 2011, 10:17.

32. Fawcett T: ROC Graphs: Notes and Practical Considerations for Researchers. Netherlands: Kluwer Academic Publishers; 2004.

33. Duffy PE, Sahu T, Akue A, Milman N, Anderson C: Pre-erythrocytic malaria vaccines: identifying the targets. Expert Rev Vaccines 2012, 11(10):1261-1280

34. Artzy-Randrup Y, Rorick MM, Day K, Chen D, Dobson AP, Pascual M: Population structuring of multi-copy, antigen-encoding genes in plasmodium falciparum. elife 2012, 1:e00093.

doi:10.1186/1471-2180-13-244

Cite this article as: Rorick et al.: Homology blocks of Plasmodium

falciparum var genes and clinically distinct forms of severe malaria in a local population. BMC Microbiology 2013 13:244.

\section{Submit your next manuscript to BioMed Central and take full advantage of:}

- Convenient online submission

- Thorough peer review

- No space constraints or color figure charges

- Immediate publication on acceptance

- Inclusion in PubMed, CAS, Scopus and Google Scholar

- Research which is freely available for redistribution 